\title{
Effectiveness of autolevellers when they used for improvement of periodic faults which may be occurred in some textile processes
}

\begin{abstract}
Autoleveler equipments are used in carding and drawing process in textile spinning industry in order to improve nonperiodic and periodic faults occurring in these or previous processes automatically. However, it was shown in the work published recently 1 that these equipments can be useless and even more disturbing in case of incorrect setting of these equipments used in the correction of the non periodic faults. This paper going one step further investigates the effectiveness of improvement of periodic faults and compares this effectiveness with that of the nonperiodic faults. The findings show that, in case of incorrect setting of the autoleveler equipments can be useless and even worse in case of non periodic faults too. But more interestingly, the findings obtained indicate that, the effectiveness of improving for periodic faults are less compare to that of nonperiodic faults nearly in any cases regardless of whatever wavelength of periodic faults are.
\end{abstract}

Keywords: automatic process control, autoleveling, dead time setting, lap setting; sliver regulation, correction of periodic faults
Volume I Issue 6 - 2017

\author{
A Baki Engin \\ Department of Industrial Engineering, University of Sakarya, \\ Turkey
}

Correspondence: A Baki Engin, Department of Industrial Engineering, University of Sakarya, Sakarya, Turkey, Email bengin@sakarya.edu.tr

Received: April 15, 2017 | Published: May 29, 2017

\section{Introduction}

The aim of spinning in textile is to produce a yarn having a suitable and constant quality with the lowest possible production costs. Today's textile spinning industries, with its high speeds and high productivity machines supervised by minimum operator and other personnel, requires, wherever possible and suitable, production and process control based on some form of technical equipments. On the other hand, process control by sampling are needed where continuous process control is not available, not possible or too costly, e.g., the testing of certain property of yarns and rovings in order to determine optimum processing conditions or for calibrating selected machines, etc. $^{2}$

Autolevelers, as one of those technical equipments used particularly in the finisher drawing passage are last opportunity to correct the faults which to degrade and perhaps to ruin the quality of large amount of subsequent unfinished and finished material such as comber silver, roving and yarn.

However, it is shown in the paper ${ }^{1}$ that in case of incorrect setting of these equipments can be useless and even disruptive for the purpose of improvement or correction of faults.

In this work, the effectiveness of improvement periodic faults by autoleveler is examined and then this effectiveness is compared with effectiveness of improvement of non periodic faults having same values of their corresponding parameters. The findings show that, in case of incorrect setting of action point, the use of autoleveler equipments is unprofitable and even disruptive as it is in non periodic faults cases. But most importantly the effectiveness of correction of periodic faults is less compared to that of non periodic faults at the same degree of incorrect setting of action point.

Yarn spinning process and importance of draw frame

Yarn spinning is shortly the joining of short fibers by drawing them from a loose fibrous mass and twisting them together. Although the yarn spinning process differs depending on spinning system it primarily involves opening, cleaning, blending, carding, drawing, prespinning (rowing), spinning, and cone winding processing stages. Spun yarns, those "composed of staple fibers held together by some binding mechanism, 'are of three types: ring spun, open-end, and airjet. ${ }^{3}$

In modern spinning process, the draw frame has an important function of the evening the slivers. However, the evenness of the slivers is essentially affected by the quality of the draft at the draw frame. There are two major causes that exert the considerable influence on sliver and yarn evenness. Firstly, the position of the draw frames in the spinning mill, which is definitively the last compensation point for correcting the faults in the slivers. Secondly, the defect produced at draw frame itself, can exert the significant disturbances and quality related problems in the further process. Material faults (e.g. short fiber contents) and machine faults (e.g. improper draft zone settings) during the drafting cause periodic and non-periodic variations (thick or thin places) in the sliver, which create problems during the subsequent process. ${ }^{4}$

\section{Periodic faults and spectrogram}

Periodic faults do not arise only drawing stage as mentioned before. This type of faults may also arise in blow room, carding, roving and spinning process due to some machinery and drafting causes. The lengths of periods can be determined on the spectrogram. Spectrograms are a sort of graphics obtained from spectrograph equipment used in spinning mills laboratories for the quality control and improvement aims. In (Figure 1) (Figure 2) are shown two spectrogram examples. In any spectrogram if the height of the peak $(\mathrm{P})$, which is named as chimney, above the basic spectrogram at any wavelength equals or over by 50 percent of height of basic spectrum at that wavelength indicates to a sufficiently serious periodic fault requiring corrective action to be taken immediately. There are 
mainly two types of spectrograms. These are chimney-type and hilltype spectrograms as shown in (Figure 1) (Figure 2) respectively. A chimney-type spectrogram, consisting of one or more "peaks" or "chimneys", as shown in Figure 1, is normally due to a mechanical fault such as eccentric roller or gear, missing or broken of teeth in gear wheels etc. The spectrogram shown in Figure 1 indicates two periodic faults having $8 \mathrm{~cm}$ and $7 \mathrm{~m}$ wavelengths.

A hill type spectrogram, on the other hand, where several adjacent peaks is noticed, is normally due to drafting waves caused by factors such as improper settings in the drafting zone, improper pressure applied by the top rollers, too many short fibres in the material, etc. The wavelength of this type faults varies over a range. Whenever there is an occurrence of a mechanical fault, it would result in a shooting up of a particular channel in the spectrogram. However, not all faults result in deterioration in the fabric quality. This is because, the extent of influence of a periodic mass variation on the fabric quality is not only dependent on the amplitude of the spectrogram peak but also on the width and type of the woven fabric, type of fibre, yarn count.etc. ${ }^{5}$

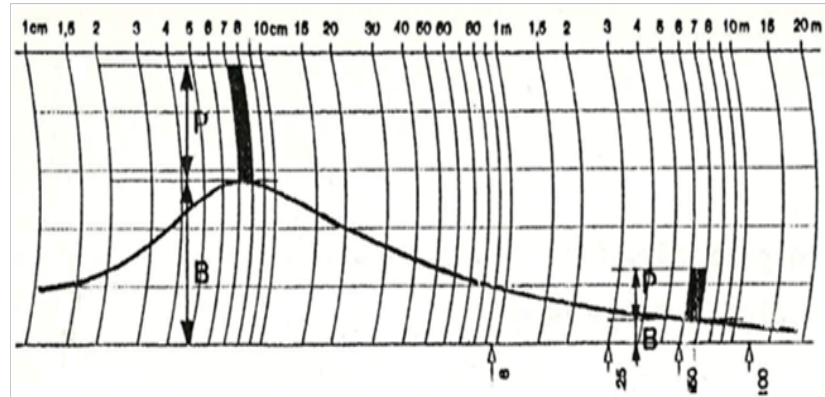

Figure I Two chimney-type periodic faults caused by mechanical fault which their lengths of waves are $8 \mathrm{~cm}$ and $7 \mathrm{~m}$ respectively.

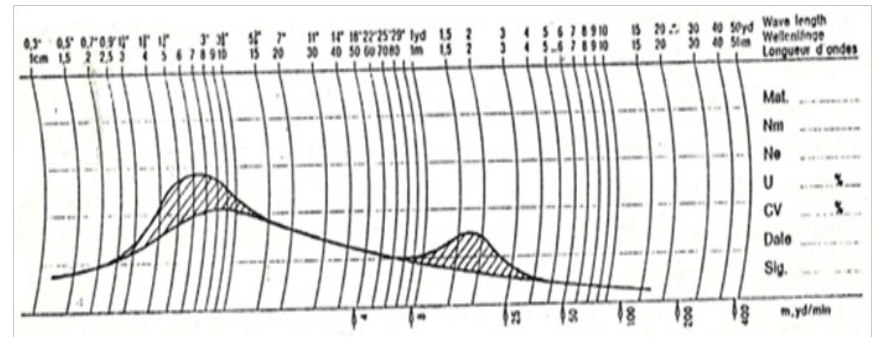

Figure 2 Two hill-types periodic faults caused by drafting fault which their average lengths of waves are around $6 \mathrm{~cm}$ and $2 \mathrm{~m}$ respectively.

\section{Autoleveling and correction of faults}

Autoleveler is described in the paper ${ }^{1}$ as "An additional device which is meant for correcting the linear density variations in the delivered sliver by changing either the main draft or break draft of the drafting system, according to the feed variation. There are two types of autoleveling systems. These are closed system and open-loop system. Most of the draw frame autolevelers are open-loop autolevelers. In open-loop autolevelers, sensing is done at the feeding end and the correction is done by changing either a break draft or main draft of the drafting system. In closed-loop system, sensing is at the delivery side and correction is done by changing either a break draft or main draft of the drafting system". In (Figure 3) (Figure 4) are shown open and closed loop autoleveler systems together. Figure 5 depicts correct and incorrect Level of Action Points (LAP) or in other words death time settings.

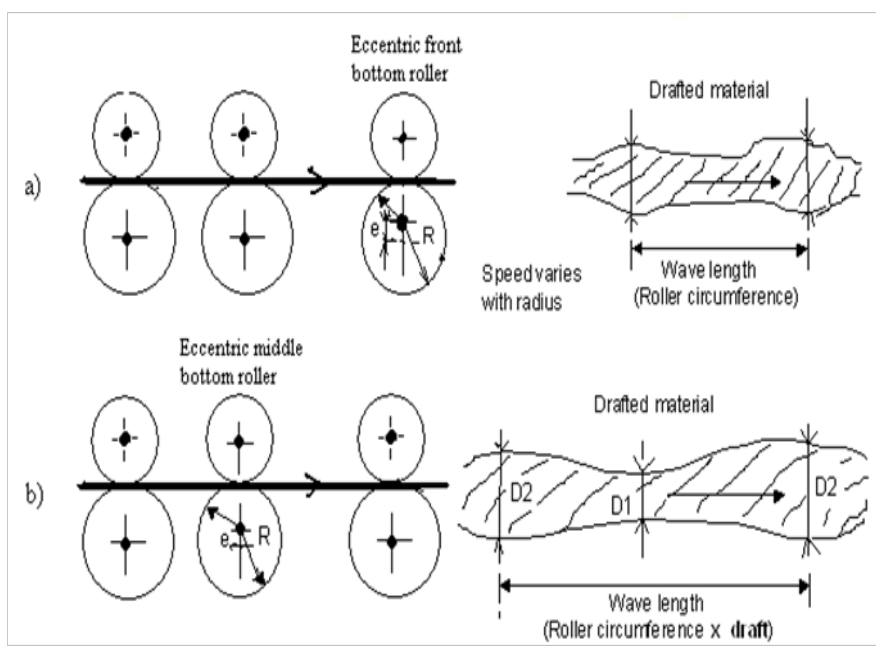

Figure 3 a) a periodic fault due to eccentric front bottom roller, b) a periodic faults due to middle bottom eccentric roller.

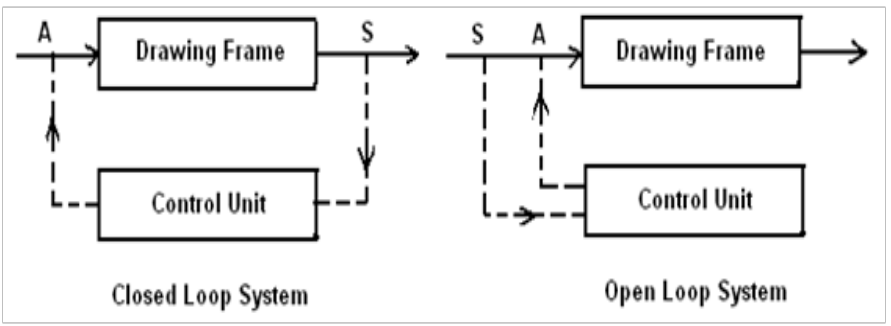

Figure 4 Open and closed loop autoleveler systems.

The distance that separates the scanning rollers and the point of action is called the zero point of regulation or LAP. This leads to the calculated correction on the corresponding defective material. Moreover, in the case of a change in fiber material, the machine settings and process controlling parameters such as production speed, material, break draft setting, main draft setting, feeding tension, and setting of the sliver guiding rollers, LAP is needed to be altered. ${ }^{4}$

\section{Materials and methods}

As expressed above, the determination of LAP correctly is very even vital important issue in order to obtain maximum benefit from autoleveler. In case of incorrect LAP setting, depending on the amount of early or late determination of action time, the effectiveness of regulation decreases. However, at least due to inevitable inertias of autoleveler components such as servomotor, differential, speed sensor and other machinery reasons, it is not possible, in any case, to obtain completely corrected, pure regular silver at the end of autoleveling process.

In the previous work (1) in case of incorrect death time setting or with alternative statement LAP setting, the effectiveness of correction of the silver having non periodic random (exponentially distributed) faults is analysed. In the present work, the analysis is taken a step further and it is examined the effectiveness of correction unevenness silver having periodic faults in case of incorrect LAP setting. The simulation approach is also used in this work considering basic assumptions and the algorithm given in that paper.

In our model, the periodic fault seen in (Figure 3a) (Figure 3b) are simplified as seen in (Figure 6a) (Figure 6b). The periodic faults 
considered in the work are chimney-type periodic faults, which occurred generally due to machinery faults or other reasons as it is pressure marks on the top rollers. The wavelengths of this type of periodic faults are same and more marked then drafting periodic faults. In the (Figure 6a) (Figure 6b), "a" and "b" represents length and period of a periodic thick place fault. The fault length "a" depends on the dimension of the defective machine part. The distance between faults " $b$ " corresponds to the circumference of the roller, e.g. at the front roller of a drafting system. For example, an eccentric front roller of draw frame leads to a periodic fault with a wavelength of $80 \mathrm{~mm}$, which this value is used in the work, as this roller always causes faulty drafts in the draw-box within the same time intervals. On the other hand, D1 and D2 have shown in the (Figure 6a) (Figure 6b) represent the diameter of normal and thick place of a periodic fault respectively.

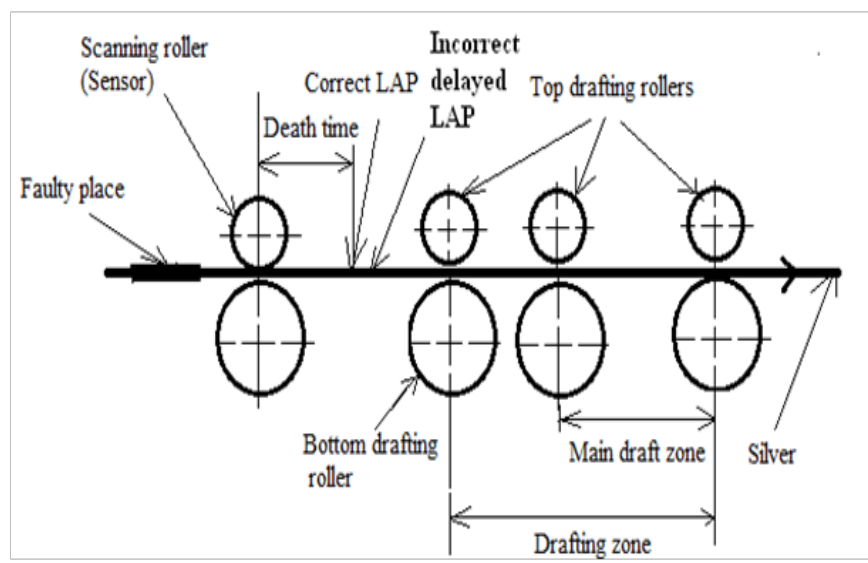

Figure 5 Improving faulty unevenness silver in draving machine by autoleveler equipment.

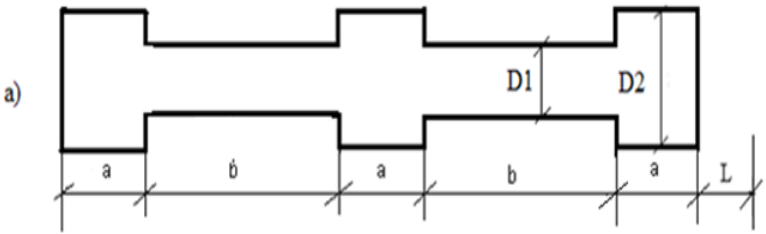

The unevennes silver, which has periodical faults, before to corrected by autoleveler

b)

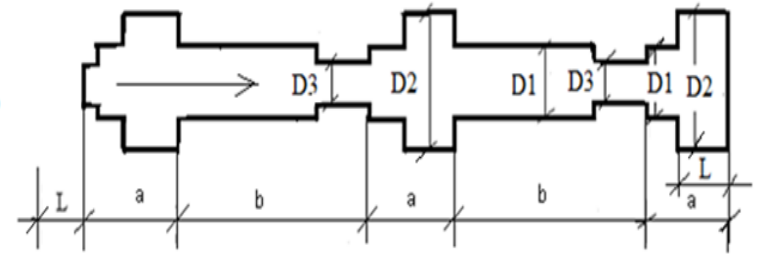

The silver after corrected by autoleveler in the case of "L " $\mathrm{mm}$ incorrect action point or death time setting

Figure 6 Unevenness silver having periodic faults before and after autoleveling.

(Figure 6a) (Figure 6b) describe the mechanism of correction by autoleveler in case of incorrect setting of LAP. As it can be seen from the Figure $6 \mathrm{a}$, if the command given by sensor as $\mathrm{L} \mathrm{mm}$ delayed, the form of the corrected unevenness silver will be like shown in the Figure $6 \mathrm{~b}$.

The graphics shown in Figures 7-24 has been obtained by means of the computer simulation program which its algorithm is given in the paper mentioned before, considering length of $135 \mathrm{~m}$ and $165 \mathrm{~m}$ simulated silver depending on parameters " $a$ " and " $b$ " considered and indicated in the related figures.

As seen from Figure $6 \mathrm{~b}$, at the beginning of correction process the silver of Lmm leaves drafting zone as uncorrected with diameter of D2 $\mathrm{mm}$ due to delayed implementation of command given as described in Figure 5. Later, diameters of the silver autoleveled change patterning as D1, D3, D1, D2, D1, D3, D1, and D2mm respectively. Here, D3 can be calculated as shown in the paper (1) as $\mathrm{D} 3=\mathrm{D} 1^{2} / \mathrm{D} 2$. In this work, $\mathrm{D} 1=24 \mathrm{~mm}, \mathrm{D} 2=32 \mathrm{~mm}$ are also taken in the model as the diameter of normal and thick places of the simplified periodic faults. In the simulation program used various length of periodic faults "a", distance between periodic faults "b" and various deviations from optimal LAP are considered as shown in Figures 7-24. The effectiveness of correction is measured decrease in percent $\mathrm{CV}$ for $10 \mathrm{~mm}$. In this work, is also examined the effectiveness of improvement of non periodic random (exponentially distributed) faults, which their average of lengths is shown in $1 / \lambda_{1}$ and average of distances between themselves in $1 / \lambda_{2}$ correspondingly.

\section{Conclusion}

The Figures 7-24 show the effectiveness of regulation of both periodic and non periodic faults by autoleveler in case of incorrect setting of LAP. It is noticed from all Figures 7-24 that the irregularity $\mathrm{CV}$ for $10 \mathrm{~mm}$ is nearly same for uncorrected silvers having both periodic and non periodic faults. Secondly, in case of in correct setting of LAP the effectiveness of correction of unevenness silver having both periodic and non periodic faults decrease depending on size and distance between faults and after from certain point the irregularity of autoleveled silver increase markedly. Thirdly, in the case of the same degree of incorrect setting, size and distance between faults, the effectiveness of correction of unevenness silver having periodic fault is less compared to that of having non periodic faults. However, periodic faults have an advantage compared to non periodic random faults so that sources of periodic faults can be found and eliminated more easily by means of spectrogram analysis.

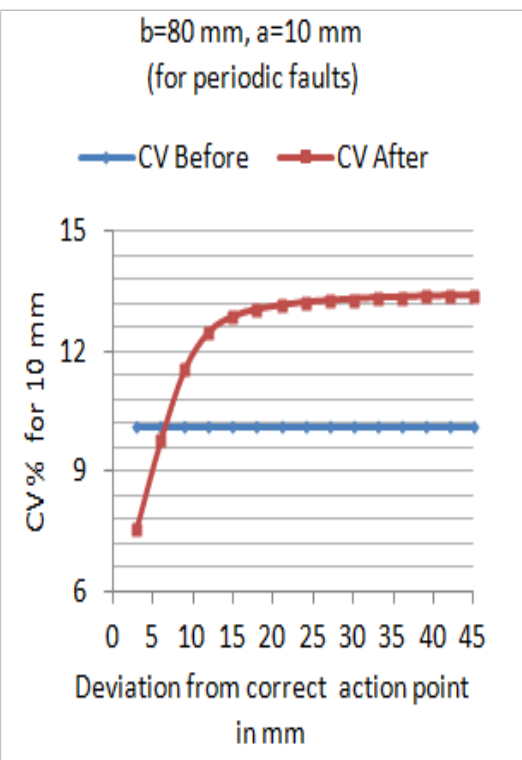

Figure 7 Effectiveness of regulation depending on LAP setting, distance between faults " $b$ " and length of fault "a". 


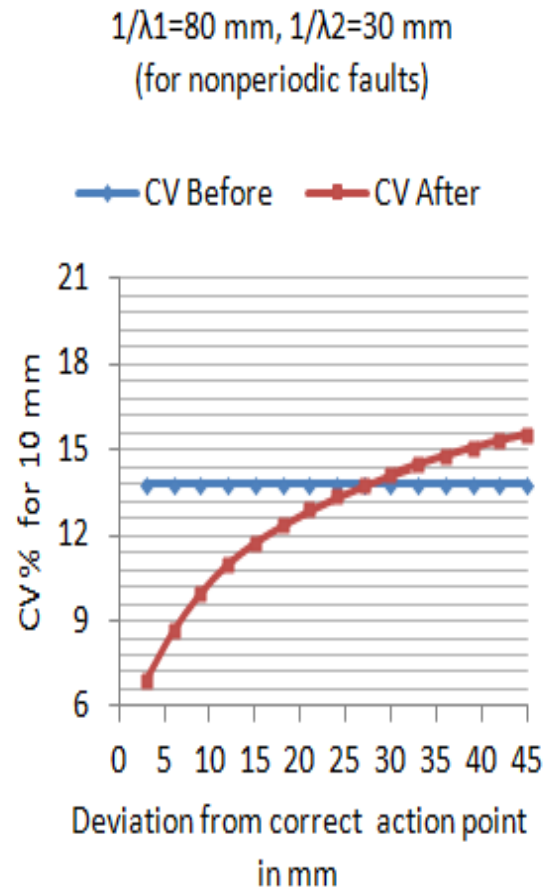

Figure 8 Effectiveness of regulation depending on LAP setting, distance between faults in average $I / \lambda_{1}$, and length of fault in average $I / \lambda_{2}$.

Therefore, the degree of benefit achieved by autoleveling equipments will be proportional to the degree of correct setting of LAP, so that, in case of improper setting of LAP or using the autoleveling equipments as malfunction, it will be better to run the draw frame without autoleveler, especially when the fault distance and length decrease.

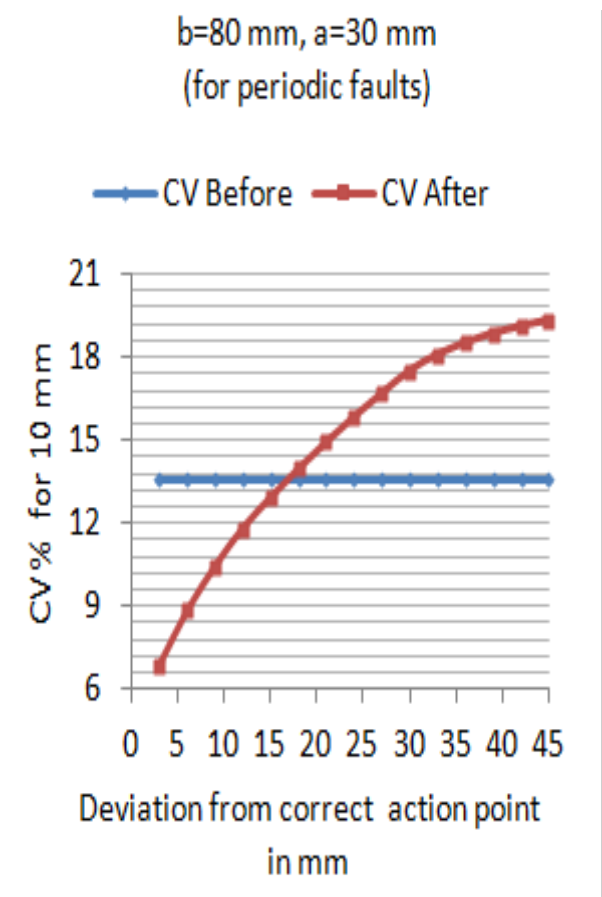

Figure 9 Effectiveness of regulation depending on LAP setting, distance between faults "b" and length of fault "a".

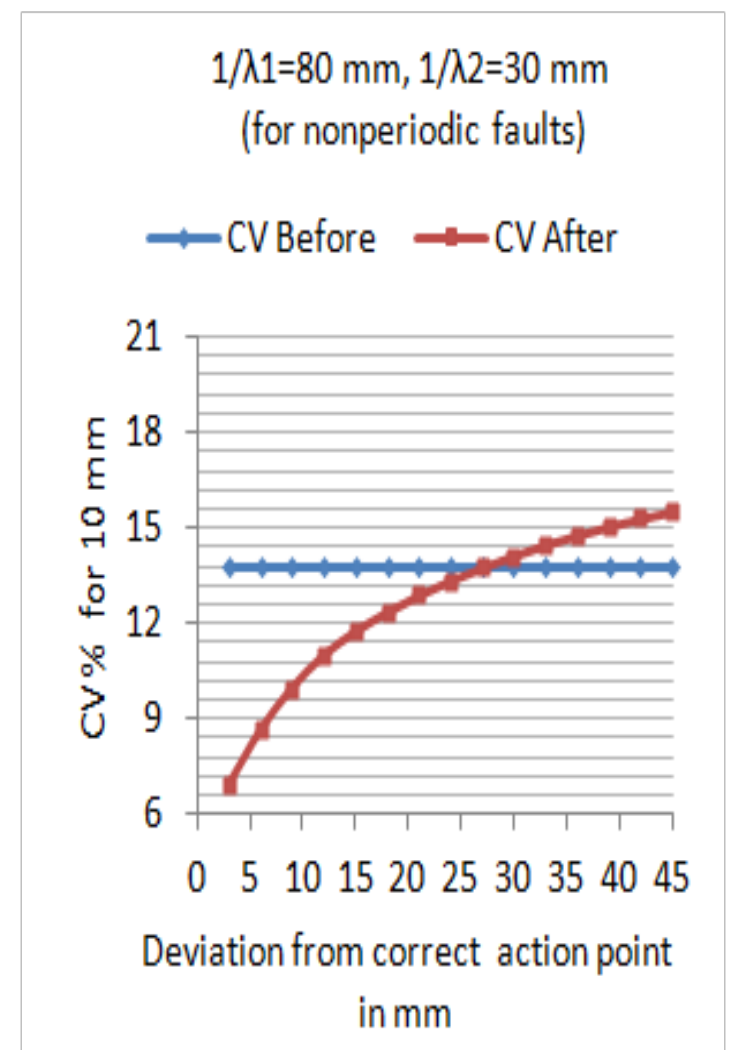

Figure 10 Effectiveness of regulation depending on LAP setting, distance between faults in average $I / \lambda_{1}$, and length of fault in average $I / \lambda_{2}$.

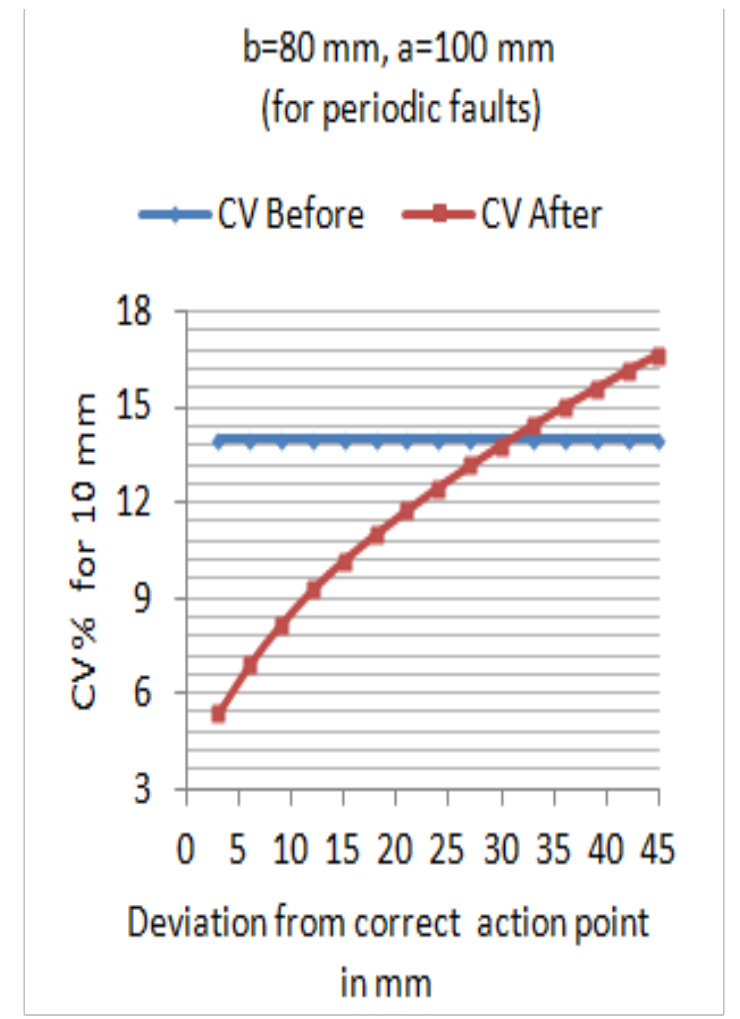

Figure II Effectiveness of regulation depending on LAP setting, distance between faults "b" and length of fault "a". 


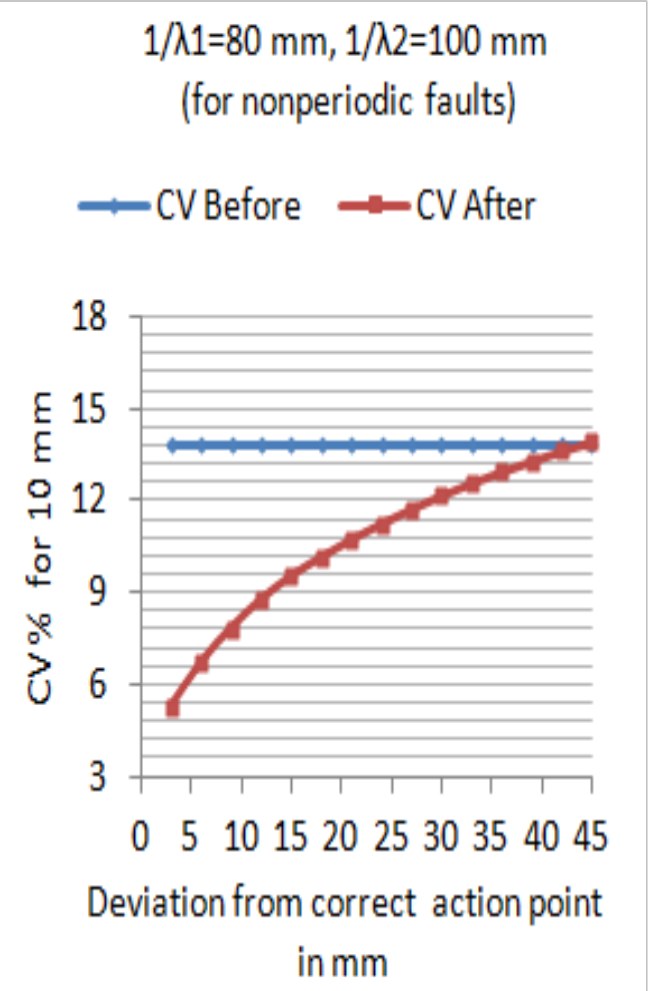

Figure 12 Effectiveness of regulation depending on LAP setting, distance between faults in average $I / \lambda_{1}$, and length of fault in average $I / \lambda_{2}$.

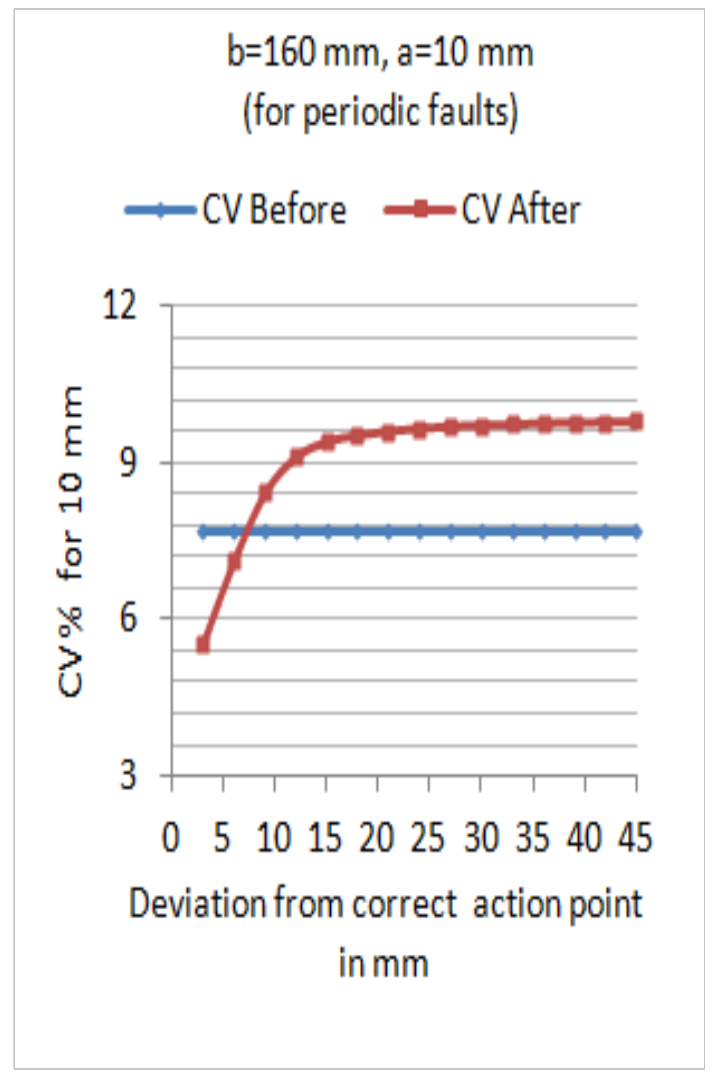

Figure 13 Effectiveness of regulation depending on LAP setting, distance between faults "b" and length of fault "a".

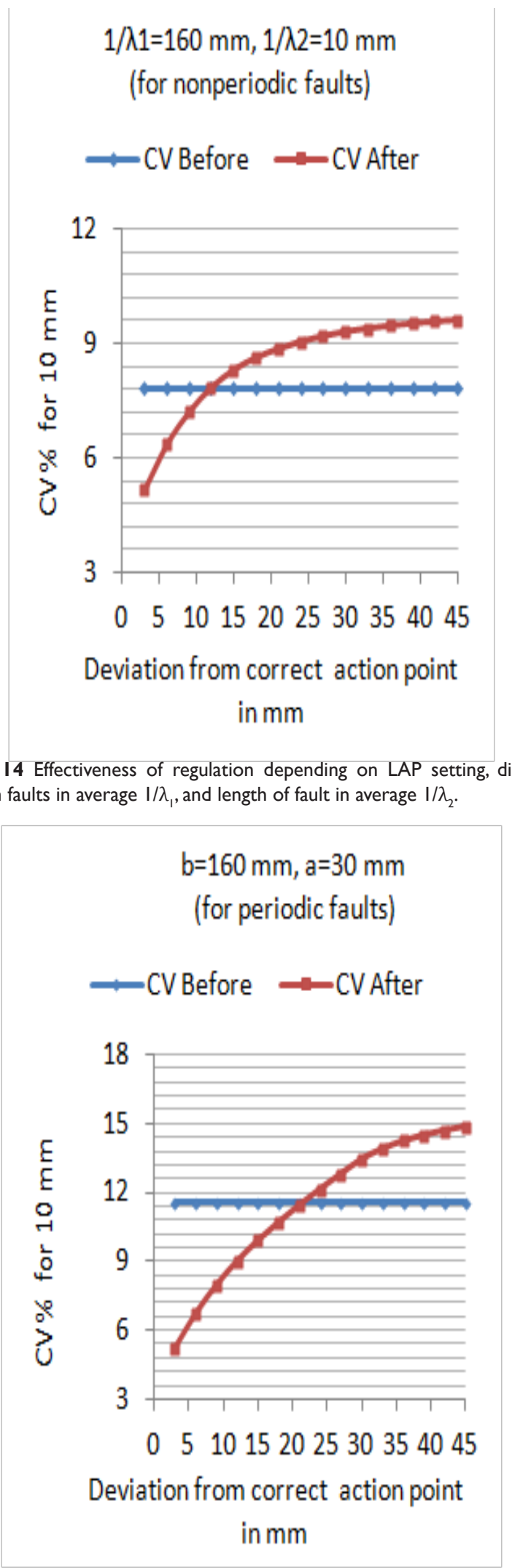

Figure 15 Effectiveness of regulation depending on LAP setting, distance between faults "b" and length of fault "a". 


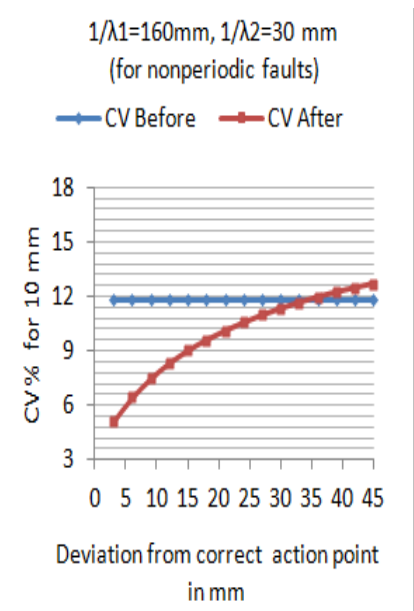

Figure 16 Effectiveness of regulation depending on LAP setting, distance between faults in average $I / \lambda_{1}$, and length of fault in average $1 / \lambda_{2}$.

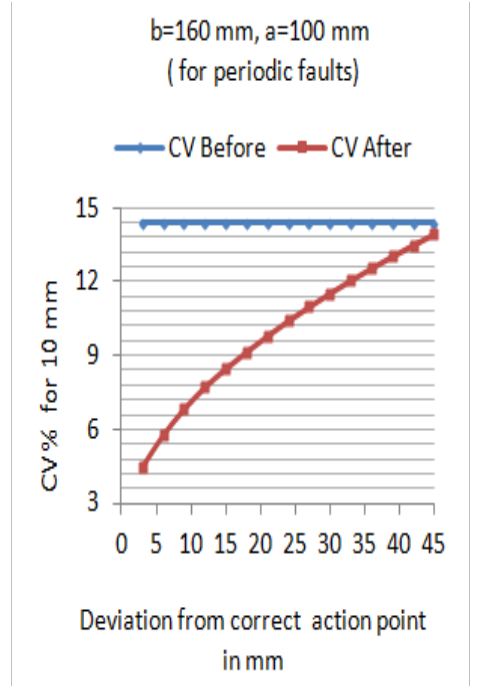

Figure 17 Effectiveness of regulation depending on LAP setting, distance between faults "b" and length of fault "a".

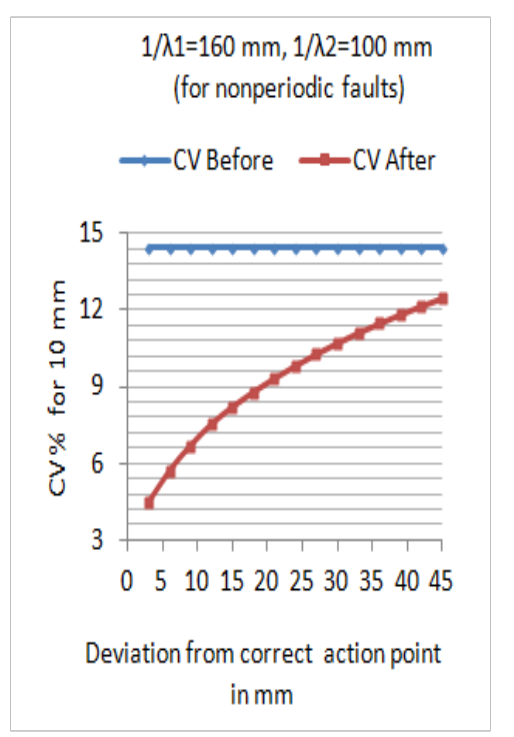

Figure 18 Effectiveness of regulation depending on LAP setting, distance between faults in average $I / \lambda_{1}$, and length of fault in average $I / \lambda_{2}$.

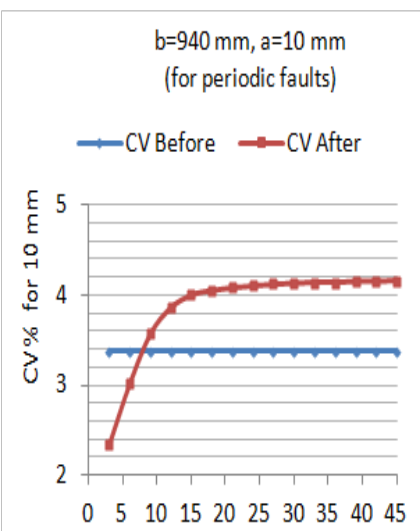

Deviation from correct action point in $\mathrm{mm}$

Figure 19 Effectiveness of regulation depending on LAP setting, distance between faults "b" and length of fault "a".

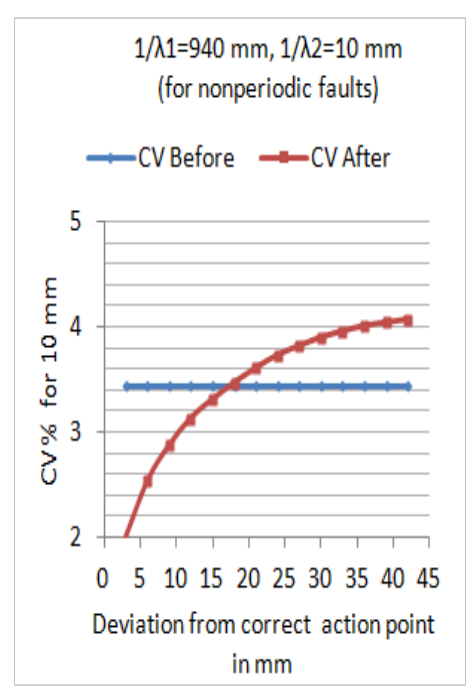

Figure 20 Effectiveness of regulation depending on LAP setting, distance between faults in average $I / \lambda_{1}$, and length of fault in average $I / \lambda_{2}$.

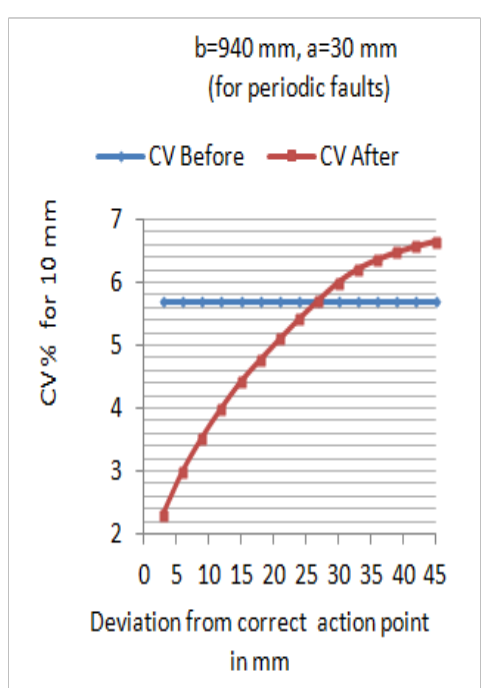

Figure 2 I Effectiveness of regulation depending on LAP setting, distance between faults "b" and length of fault "a". 


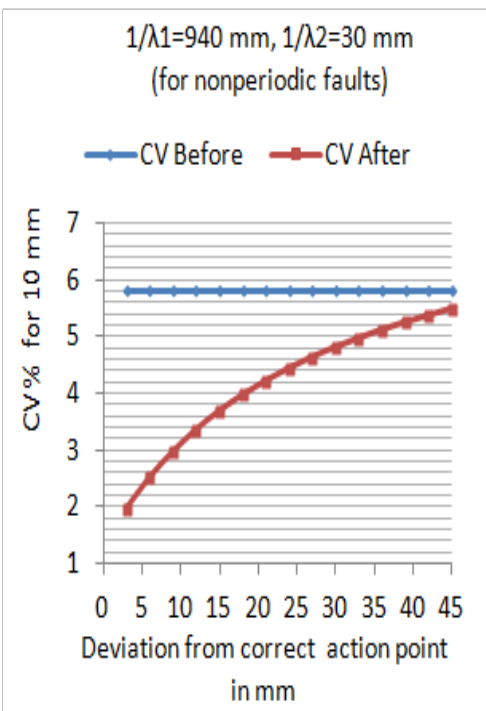

Figure 22 Effectiveness of regulation depending on LAP setting, distance between faults in average $I / \lambda_{1}$, and length of fault in average $I / \lambda_{2}$.

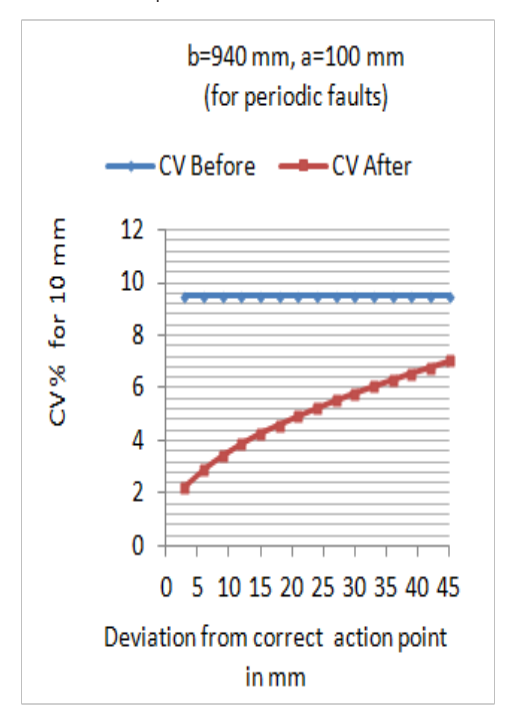

Figure 23 Effectiveness of regulation depending on LAP setting, distance between faults " $b$ " and length of fault "a".

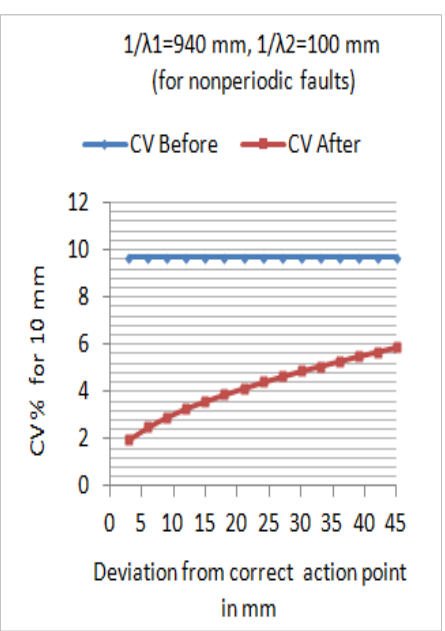

Figure 24 Effectiveness of regulation depending on LAP setting, distance between faults in average $I / \lambda_{1}$, and length of fault in average $I / \lambda_{2}$.

\section{Acknowledgements}

None.

\section{Conflict of interest}

Author declares there is no conflict of interest in publishing the article.

\section{References}

1. Engin AB. The effect of inappropriate dead time settings on the regulation of unevenness of material implemented by the automatic control: an application in the textile spinning industry. $J$ Textile Institute. 2016;107(11):1442-1449.

2. Zellweger Uster. Sliver data for the processes prior to spinning. Uster News Bulletin. 1986.

3. Engin AB. An Application and Use of Economic and Statistical Control Chart Design for the Textile Yarn Industry. Quality Engineering. 2004;16(4):625-635.

4. Farooq A, Cherif C. Use of artificial neural networks for determining the levelling action point at the auto-leveling draw frame. Textile Research J. 2008;78(6):502-509.

5. Thilagavathi G, Karthik T. Process Control and Yarn Quality in Spinning. Woodhead Publishing; 2015. p. 424. 